\section{Mortalidade materna no Brasil: o que mostra a produção científica nos últimos 30 anos?}

\author{
Maternal mortality in Brazil: what has the scientific \\ literature shown in the last 30 years?
}

\footnotetext{
1 Instituto de Saúde da Comunidade, Universidade Federal Fluminense, Niterói, Brasil.

2 Secretaria Estadual de Saúde e Defesa Civil do Rio de Janeiro, Niterói, Brasil. 3 Faculdade de Medicina, Universidade Federal Fluminense, Niterói, Brasil.

Correspondência M. L. Morse

Programa de Pós-graduação em Saúde Coletiva, Instituto de Saúde da Comunidade, Universidade Federal Fluminense.

Av. Marquês do Paraná 303, 4o andar (Prédio anexo ao HUAP), Niterói, RJ 24130-210, Brasil. marcialait@bol.com.br
}

\begin{abstract}
The aim of this study was to analyze maternal mortality in Brazil in the last 30 years, by means of a literature review. The authors performed an electronic search of scientific articles from 1980 to 2010 in LILACS and MEDLINE and found 486 abstracts, of which 50 articles were selected. Studies showed a decrease in the maternal mortality ratio (MMR), although varying across regions of the country. A few articles evaluated maternal mortality factors, identifying social inequalities associated with skin color and schooling. There was persistent underreporting of maternal deaths and inadequate completion of death certificates. Direct obstetric causes were the most frequent, mainly hypertensive diseases of pregnancy. Analysis of avoidability revealed deficiencies in prenatal and childbirth care. Despite the relevance of maternal mortality in Brazil, there are few studies on the subject. Although MMR has decreased, it is still above the desired levels. Improvements are thus needed in the quality of prenatal and perinatal care.
\end{abstract}

Maternal Mortality; Mortality Rate; Review

\author{
Marcia Lait Morse 1,2 \\ Sandra Costa Fonseca 1 \\ Mariane Doelinger Barbosa ${ }^{3}$ \\ Manuele Bonatto Calil 3 \\ Fernanda Pinella Carvalhal Eyer 3
}

\section{Introdução}

A mortalidade materna é um grave problema de saúde pública, notadamente nos países em desenvolvimento, onde ocorrem $99 \%$ dos óbitos maternos 1. Segundo a Organização Mundial da Saúde (OMS), a razão de mortalidade materna (RMM) no ano de 2008 variou de 3/100.000 nascidos vivos (NV) na Irlanda a 1.200/100.000 NV no Afeganistão.

Em 2008, a Organização Pan-Americana da Saúde (OPAS) avaliou a situação de saúde da Região das Américas 2: a RMM variou de 5,9/100.000 NV no Canadá a 690/100.000 NV no Haiti, refletindo a extrema desigualdade socioeconômica da região.

Quanto às causas dos óbitos maternos, também se observam diferenças de acordo com o grau de desenvolvimento dos países. Khan et al. 3 apontaram que cerca de metade das mortes na América Latina e Caribe é por distúrbios hipertensivos e hemorragias, com um percentual importante de não-definidas. Já nos países desenvolvidos, causas diretas relacionadas à anestesia e à cesariana são as mais frequentes 3 .

Na Cúpula do Milênio da Organização das Nações Unidas (ONU), foram adotadas oito Metas de Desenvolvimento, entre elas, reduzir a mortalidade materna em $75 \%$ até 2015 . Segundo a ONU 4 , a mortalidade materna diminuiu menos de $1 \%$ ao ano até 2005 , abaixo dos $5,5 \%$ anuais necessários para atingir a meta. 
No último relatório da OMS sobre mortalidade materna, o Brasil teve posição abaixo da meta do milênio; nos últimos 18 anos alcançou redução de $52 \%$ (120 por $100.000 \mathrm{NV}$ em 1990, 64/100.00 NV em 2005 e 58/100.000 NV em 2008), com velocidade média anual de queda de $4 \%$, quando o ideal seriam $5,5 \% 1$. Os dados nacionais, no entanto, mostram em 2006 e 2007 valores maiores que os apontados pela OMS, com RMM de 77,00/100.000 NV (Ministério da Saúde; http://tabnet.datasus.gov.br/cgi/idb2009/C03b. htm, acessado em Nov/2010), confirmando a avaliação da OMS de "deficiência de registro completo e adequado, mas com outros tipos de dados disponíveis" - uma posição intermediária em termos de qualidade dos dados 1 .

Problemas de notificação e qualidade do preenchimento de óbitos maternos têm sido apontados já há alguns anos no Brasil 5 . Um estudo de 1999 em 15 municípios brasileiros 6 identificou sub-registro de $8,7 \%$ e subinformação de $130 \%$, obtendo-se um fator de ajuste da RMM de 2,3. Outro estudo 7, sobre o preenchimento da Declaração de Óbito (DO) nos campos 43 e 44, que informam status e momento do óbito em relação à gravidez, apontou informação ignorada superior a $87 \%$ para estas variáveis.

O Estudo da Mortalidade de Mulheres de 10 a 49 Anos, com Ênfase na Mortalidade Materna 8 identificou a necessidade de ajuste para RMM. Baseado nos dados originais das capitais brasileiras de 2002, a mortalidade materna, que era de 38,9 por $100.000 \mathrm{NV}$, foi corrigida para 54,3 por $100.000 \mathrm{NV}$. Portanto, o fator de ajuste foi 1,4. Como a pesquisa se restringiu às capitais, que somam $26,6 \%$ do total de mortalidade em idade fértil no país, esse fator é o mínimo a ser considerado 8 .

Os dados recentes dos sistemas de informação no Brasil apontaram aumento da RMM 4; que, em parte, pode ser pela melhoria dos registros e captação de óbitos, a partir da criação dos Comitês de Mortalidade Materna ${ }^{9}$ e da adoção do fator de ajuste.

Os dados também sugerem valores mais elevados nas mulheres de menor renda e escolaridade, da raça negra e com pouco acesso à assistência, sendo um importante indicador das condições de vida e injustiça social 5 .

Nesse contexto, como a pesquisa científica tem contribuído para o melhor conhecimento da mortalidade materna no nosso país? Tem sido possível superar a subnotificação e conhecer a real magnitude do problema? As causas proximais e os determinantes socioeconômicos têm sido investigados? Que aspectos da assistência à saúde da mulher têm sido explorados? Existem diferenças regionais?
Embora seja um dos problemas mais relevantes de saúde pública, não há revisões da produção científica brasileira acerca da mortalidade materna.

O objetivo deste trabalho foi conhecer o perfil epidemiológico, variações temporais e regionais da mortalidade materna no Brasil, por meio de uma revisão da literatura, identificando estudos sobre o tema publicados em periódicos nacionais ou internacionais.

\section{Metodologia}

Foram consultadas as seguintes bases bibliográficas: LILACS, via Biblioteca Virtual de Saúde (BVS); e MEDLINE, via PubMed. Os descritores e respectivos termos utilizados nas buscas foram: na base MEDLINE - maternal mortality AND Brazil; na base BVS - mortalidade materna AND Brasil. Também foram usados os nomes dos estados e regiões brasileiras (Norte, Nordeste, Centro-oeste, Sudeste, Sul): mortalidade materna AND nome do estado; mortalidade materna AND nome da região, para aumentar a sensibilidade da busca.

O período de publicação foi de 1980 a fevereiro de 2010, sem restrição de idioma. Tal período foi definido porque a implantação dos comitês de morte materna iniciou-se na década de 80.

Foram incluídos estudos descritivos ou analíticos, dos quais fosse possível retirar dados quantitativos sobre mortalidade materna, suas causas básicas e determinantes. Excluíram-se relatos de casos, teses, relatórios, pesquisas qualitativas e artigos que explorassem exclusivamente métodos de estudos sobre o tema.

Foram priorizados estudos populacionais, com exclusão daqueles limitados a unidades hospitalares, que não caracterizassem base populacional. Também foram excluídos estudos nos quais os óbitos foram analisados por local de ocorrência e não de residência, o que não permitiria caracterizar a RMM de base populacional.

A busca, leitura dos artigos e extração dos dados foi realizada sempre por pelo menos três pesquisadores: as autoras M.L.M. e S.C.F. e estudantes de Medicina do nono período. A autora principal (M.L.M.) releu todos os artigos para avaliar a seleção.

Não foi aplicado qualquer escore de avaliação sobre a qualidade dos artigos pela inexistência deste tipo de instrumento para estudos de cunho descritivo. 


\section{Resultados}

Foram identificados 163 resumos elegíveis na busca via BVS (com a palavra-chave Brasil). No levantamento adicional por estados e regiões, mais 219 resumos. Na busca pelo PubMed 104 resumos foram elegíveis.

Após aplicação dos critérios de inclusão e exclusão, e da identificação de duplicidades, restaram 47 artigos. Foram captados mais três artigos nas referências bibliográficas dos 47 encontrados, totalizando 50 artigos selecionados (Figura 1). Dezoito (36\%) foram publicados em periódicos de Saúde Pública, 18 (36\%) em publicações de ginecologia e obstetrícia e os demais em revistas de enfermagem ou clínica.
A Tabela 1 apresenta os estudos sobre mortalidade materna, por regiões, em ordem cronológica (ano da coleta de dados), para mostrar a evolução em nosso país ao longo de 30 anos. AS RMM estão apresentadas como RMM-1 - estimativa bruta, sem correção - e RMM-2, após ajuste pelo fator de correção usado pelos autores.

Dos artigos selecionados, 7 (14\%) apresentavam dados de abrangência nacional, dos quais 2 também dados regionais. A Região Sudeste apresentou o maior volume dessa produção, $46 \%(23 / 50)$ dos artigos. A Região Sul teve $26 \%$ (13/50), a Região Nordeste $14 \%$ (7/50), enquanto que as regiões Centro-oeste e Norte contribuíram com 1 artigo cada (2\%).

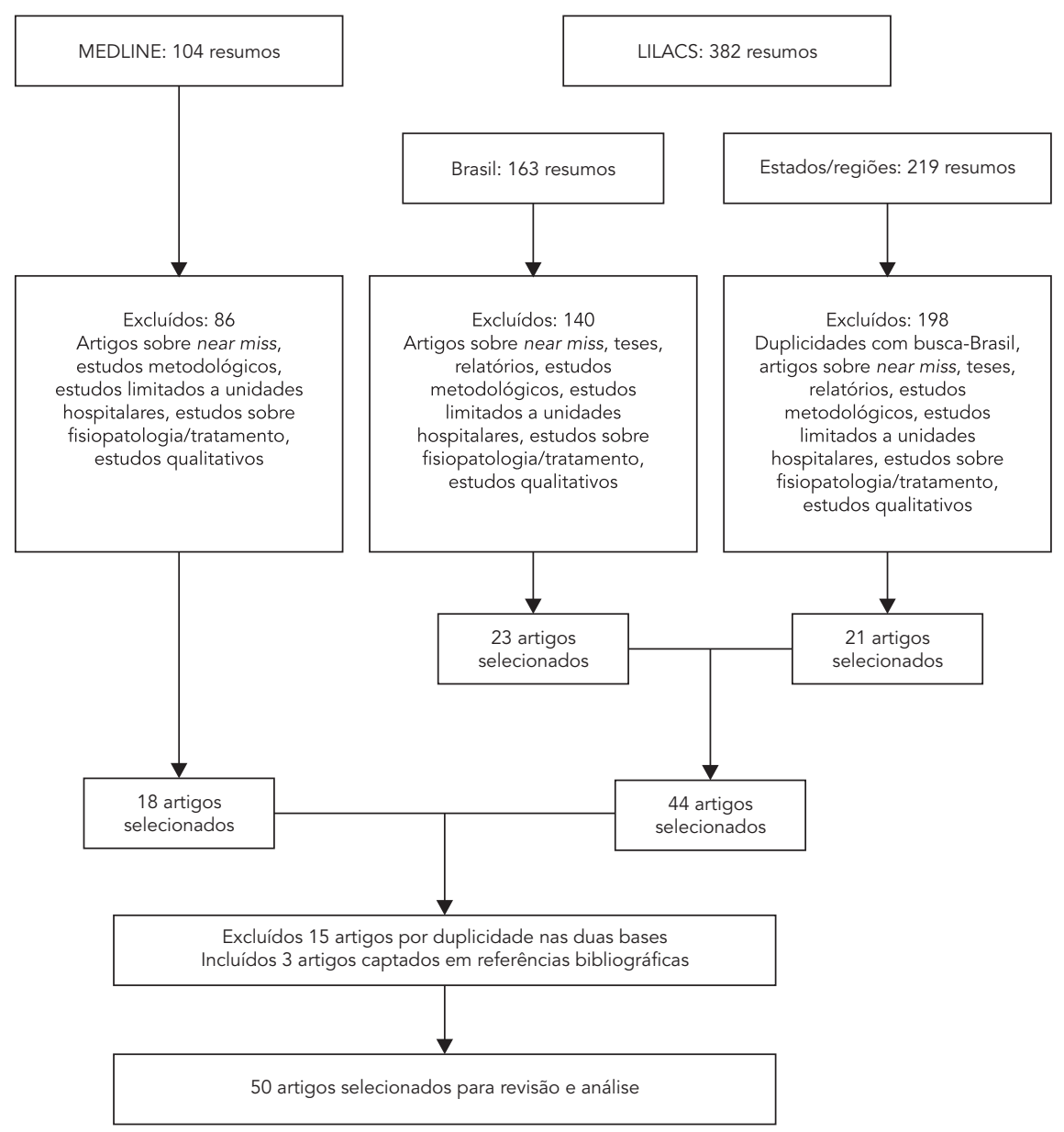


Tabela 1

Estudos sobre mortalidade materna no Brasil.

\begin{tabular}{|c|c|c|c|c|c|c|c|}
\hline Autor & $\begin{array}{l}\text { Período } \\
\text { estudado }\end{array}$ & Local & População & Métodos e fonte dos dados & Causa principal & RMM1 & RMM2 \\
\hline Brasil & & & & & & & \\
\hline $\begin{array}{l}\text { Siqueira } \\
\text { et al. } 10\end{array}$ & 1980 & Regiões & $\begin{array}{l}\text { Todas } \\
\text { as faixas } \\
\text { etárias }\end{array}$ & $\begin{array}{c}\text { Revisão dos dados oficiais: } \\
\text { Anuário Estatístico do Brasil e } \\
\text { Censo de } 1980\end{array}$ & Hipertensão & 70 & 154,3 \\
\hline $\begin{array}{l}\text { Siqueira \& } \\
\text { Tanaka } 11\end{array}$ & 1980 & Brasil & 10-19 anos & $\begin{array}{l}\text { Revisão dos dados oficiais: } \\
\text { estatísticas de mortalidade e } \\
\text { Censo de } 1980\end{array}$ & Hipertensão & $15-19$ anos; 64,3 & \\
\hline Victora 12 & $\begin{array}{l}1995- \\
1997\end{array}$ & Brasil e regiões & 10-49 anos & $\begin{array}{c}\text { Revisão dos óbitos registrados } \\
\text { no SIM }\end{array}$ & Hipertensão & 44 & 140 \\
\hline $\begin{array}{l}\text { Sousa } \\
\text { et al. } 13\end{array}$ & 2002 & $\begin{array}{c}\text { Brasil (26 } \\
\text { capitais e DF) }\end{array}$ & 10-49 anos & $\begin{array}{c}\text { Linkage de sistemas de } \\
\text { informação: SIM, SINASC e } \\
\text { SIH-SUS }\end{array}$ & Não descrita & 46,4 & 64,9 \\
\hline $\begin{array}{l}\text { Laurenti } \\
\text { et al. } 14,15\end{array}$ & 2002 & $\begin{array}{l}15 \text { capitais } \\
\text { brasileiras }\end{array}$ & 10-49 anos & RAMOS & Hipertensão & 38,9 & 54,3 \\
\hline Martins 16 & 2002 & $\begin{array}{c}\text { Capitais e } \\
\text { outras cidades }\end{array}$ & $10-49$ anos & $\begin{array}{c}\text { Revisão de óbitos e dados } \\
\text { oficiais: DO, bases de dados dos } \\
\text { Comitês de Mortalidade Materna } \\
\text { e teses }\end{array}$ & Hipertensão & $\begin{array}{c}\text { Pretas: } 227,6 / \text { Brancas: } \\
48,7\end{array}$ & \\
\hline Sudeste & & & & & & & \\
\hline $\begin{array}{l}\text { Siqueira } \\
\text { et al. } 10\end{array}$ & 1980 & $\begin{array}{l}\text { Estado de São } \\
\text { Paulo }\end{array}$ & $\begin{array}{l}\text { Todas } \\
\text { as faixas } \\
\text { etárias }\end{array}$ & $\begin{array}{l}\text { Revisão dos dados oficiais: } \\
\text { CIS/SEADE }\end{array}$ & Hipertensão & 52 & 58 \\
\hline $\begin{array}{l}\text { Tanaka } \\
\text { et al. } 17\end{array}$ & $\begin{array}{l}1980- \\
1984\end{array}$ & $\begin{array}{l}\text { Estado de São } \\
\text { Paulo }\end{array}$ & $\begin{array}{l}\text { Todas } \\
\text { as faixas } \\
\text { etárias }\end{array}$ & $\begin{array}{l}\text { Revisão dos dados oficiais: } \\
\text { CIS/SEADE }\end{array}$ & Hipertensão & & $\begin{array}{c}55,4 \\
(1980) / 48,6 \\
(1984)\end{array}$ \\
\hline Laurenti 18 & $\begin{array}{l}1960- \\
1990\end{array}$ & $\begin{array}{l}\text { Estado de São } \\
\text { Paulo }\end{array}$ & $\begin{array}{l}\text { Todas } \\
\text { as faixas } \\
\text { etárias }\end{array}$ & $\begin{array}{c}\text { Investigação de amostra de } \\
\text { declarações de óbito junto aos } \\
\text { familiares, hospitais e dados de } \\
\text { estudos de 1962-1963, } \\
1974-1975,1986\end{array}$ & Hipertensão & $\begin{array}{c}51,4(\operatorname{anos} 80) / 47,2 \\
(\operatorname{anos} 90)\end{array}$ & \\
\hline $\begin{array}{l}\text { Haddad \& } \\
\text { Silva } 19\end{array}$ & $\begin{array}{l}1991- \\
1995\end{array}$ & $\begin{array}{l}\text { Estado de São } \\
\text { Paulo }\end{array}$ & $15-49$ anos & Revisão de dados do SIM & Hipertensão & $43,7-49,6$ & \\
\hline $\begin{array}{l}\text { Laurenti et } \\
\text { al. } 20,21\end{array}$ & 1986 & São Paulo & 10-49 anos & $\begin{array}{l}\text { Investigação de } 25 \% \text { dos óbitos } \\
\text { de MIF: DO, visita domiciliar, } \\
\text { revisão de prontuários e laudos } \\
\text { de necropsias }\end{array}$ & Hipertensão & 44,5 & 99,6 \\
\hline $\begin{array}{l}\text { Boyacivan } \\
\text { et al. } 22\end{array}$ & $\begin{array}{l}1993- \\
1995\end{array}$ & São Paulo & $10-49$ anos & $\begin{array}{l}\text { Investigação dos óbitos de MIF } \\
\text { com morte materna declarada } \\
\text { ou presumível: DO, visita } \\
\text { domiciliar e hospitalar e laudos } \\
\text { de necropsias }\end{array}$ & $\begin{array}{l}\text { Hemorragias e } \\
\text { Hipertensão }\end{array}$ & & 50,2 \\
\hline
\end{tabular}

(continua) 


\begin{tabular}{|c|c|c|c|c|c|c|c|}
\hline Autor & $\begin{array}{l}\text { Período } \\
\text { estudado }\end{array}$ & Local & População & Métodos e fonte dos dados & Causa principal & RMM1 & RMM2 \\
\hline \multicolumn{8}{|l|}{ Sudeste } \\
\hline $\begin{array}{l}\text { Marcus } \\
\text { et al. } 23\end{array}$ & 1995 & São Paulo & $10-49$ anos & $\begin{array}{l}\text { Investigação dos óbitos de MIF } \\
\text { com morte materna declarada } \\
\text { ou presumível: DO, visitas } \\
\text { domiciliar e hospitalar e laudos } \\
\text { de necropsias }\end{array}$ & Hipertensão & & 51,3 \\
\hline $\begin{array}{l}\text { Pazero } \\
\text { et al. } 24\end{array}$ & 1996 & São Paulo & $10-49$ anos & $\begin{array}{l}\text { Investigação dos óbitos de MIF } \\
\text { com morte materna declarada } \\
\text { ou presumível: DO, visitas } \\
\text { domiciliar e hospitalar e laudos } \\
\text { de necropsias }\end{array}$ & Hipertensão & & 48,3 \\
\hline $\begin{array}{l}\text { Vega } \\
\text { et al. } 25\end{array}$ & 1997 & São Paulo & $10-49$ anos & $\begin{array}{l}\text { Investigação dos óbitos de MIF } \\
\text { com morte materna declarada } \\
\text { ou presumível: DO, visitas } \\
\text { domiciliar e hospitalar e laudos } \\
\text { de necropsias }\end{array}$ & Hipertensão & & 61,1 \\
\hline $\begin{array}{l}\text { Pazero et } \\
\text { al. } 26\end{array}$ & 1998 & São Paulo & $10-49$ anos & $\begin{array}{l}\text { Investigação dos óbitos de MIF } \\
\text { com morte materna declarada } \\
\text { ou presumível: DO, visitas } \\
\text { domiciliar e hospitalar e laudos } \\
\text { de necropsias }\end{array}$ & Infecção & & 58,3 \\
\hline $\begin{array}{l}\text { Vega et } \\
\text { al. } 27\end{array}$ & 1999 & São Paulo & $10-49$ anos & $\begin{array}{l}\text { Investigação dos óbitos de MIF } \\
\text { com morte materna declarada } \\
\text { ou presumível: DO, visitas } \\
\text { domiciliar e hospitalar e laudos } \\
\text { de necropsias }\end{array}$ & Hipertensão & & 58,2 \\
\hline $\begin{array}{l}\text { Vega et } \\
\text { al. } 28\end{array}$ & $\begin{array}{l}1995- \\
1999\end{array}$ & São Paulo & $10-49$ anos & $\begin{array}{l}\text { Investigação dos óbitos de MIF } \\
\text { com morte materna declarada } \\
\text { ou presumível: DO, visitas } \\
\text { domiciliar e hospitalar e laudos } \\
\text { de necropsias }\end{array}$ & $\begin{array}{l}\text { Óbitos por } \\
\text { hipertensão }\end{array}$ & 37,2 & 48 * \\
\hline $\begin{array}{l}\text { Cecatti et } \\
\text { al. } 29,30\end{array}$ & $\begin{array}{l}1985- \\
1991\end{array}$ & $\begin{array}{c}\text { Campinas (São } \\
\text { Paulo) }\end{array}$ & 10-49anos & $\begin{array}{l}\text { Investigação dos óbitos de MIF } \\
\text { com morte materna declarada } \\
\text { ou presumível: DO, visitas } \\
\text { domiciliar e hospitalar e laudos } \\
\text { de necropsias }\end{array}$ & Hipertensão & 28,6 & 45,5 \\
\hline $\begin{array}{l}\text { Parpinelli } \\
\text { et al. } 31,32\end{array}$ & $\begin{array}{l}1992- \\
1994\end{array}$ & $\begin{array}{c}\text { Campinas (São } \\
\text { Paulo) }\end{array}$ & $10-49$ anos & $\begin{array}{l}\text { Investigação dos óbitos de MIF } \\
\text { com morte materna declarada } \\
\text { ou presumível: DO, visitas } \\
\text { domiciliar e hospitalar e laudos } \\
\text { de necropsias }\end{array}$ & Aborto & 25,3 & 42,2 \\
\hline $\begin{array}{l}\text { Rezende } \\
\text { et al. } 34\end{array}$ & 1997 & $\begin{array}{c}\text { Uberlândia } \\
\text { (Minas Gerais) }\end{array}$ & $10-49$ anos & RAMOS & Hipertensão & 22,2 & 44,4 \\
\hline $\begin{array}{l}\text { Matias et } \\
\text { al. } 33\end{array}$ & $\begin{array}{l}1999- \\
2006\end{array}$ & $\begin{array}{l}\text { Jundiaí (São } \\
\text { Paulo) }\end{array}$ & $10-49$ anos & RAMOS & Hipertensão & 14,7 & 29,4 \\
\hline
\end{tabular}

(continua) 


\begin{tabular}{|c|c|c|c|c|c|c|c|}
\hline Autor & $\begin{array}{l}\text { Período } \\
\text { estudado }\end{array}$ & Local & População & Métodos e fonte dos dados & Causa principal & RMM1 & RMM2 \\
\hline \multicolumn{8}{|l|}{ Sudeste } \\
\hline Silva 35 & $\begin{array}{l}1977- \\
1987\end{array}$ & $\begin{array}{c}\text { Estado do Rio } \\
\text { de Janeiro }\end{array}$ & $10-49$ anos & $\begin{array}{c}\text { Revisão de dados do SIM: DO } \\
\text { e estatísticas de mortalidade } \\
\text { (Ministério da Saúde) }\end{array}$ & Hipertensão & $111(1977) / 56(1988)$ & \\
\hline $\begin{array}{l}\text { Theme- } \\
\text { Filha } \\
\text { et al. } 36\end{array}$ & $\begin{array}{l}1993- \\
1996\end{array}$ & Rio de Janeiro & $10-49$ anos & $\begin{array}{c}\text { Revisão de dados do SIM: DO } \\
\text { e estatísticas de mortalidade } \\
\text { (Ministério da Saúde) }\end{array}$ & Hipertensão & 57,5 & \\
\hline $\begin{array}{l}\text { Melo \& } \\
\text { Knupp } 37\end{array}$ & $\begin{array}{l}1996- \\
2004\end{array}$ & Rio de Janeiro & $10-49$ anos & SIM/SINASC & Hipertensão & $55(1996) / 69$ (2004) & \\
\hline $\begin{array}{l}\text { Kale \& } \\
\text { Costa } 38\end{array}$ & $\begin{array}{l}2000- \\
2003\end{array}$ & Rio de Janeiro & 10-49 anos & $\begin{array}{l}\text { Revisão de dados do SIM e do } \\
\text { Comitê de Mortalidade Materna }\end{array}$ & Hipertensão & 59,5 & \\
\hline Sul & & & & & & & \\
\hline Madi 39 & $\begin{array}{l}1979- \\
1982\end{array}$ & $\begin{array}{l}\text { Rio Grande } \\
\text { do Sul }\end{array}$ & & $\begin{array}{l}\text { Revisão das declarações dos } \\
\text { óbitos maternos declarados }\end{array}$ & Hipertensão & $\approx 60$ & \\
\hline $\begin{array}{l}\text { Ramos } \\
\text { et al. } 40\end{array}$ & $\begin{array}{l}1978- \\
1988\end{array}$ & $\begin{array}{l}\text { Rio Grande } \\
\text { do Sul }\end{array}$ & $15-49$ anos & $\begin{array}{l}\text { Revisão das declarações dos } \\
\text { óbitos maternos declarados }\end{array}$ & Hipertensão & $61,3(1978) / 21,9(1988)$ & \\
\hline $\begin{array}{l}\text { Riquinho \& } \\
\text { Correia } 41\end{array}$ & $\begin{array}{l}1999- \\
2001\end{array}$ & $\begin{array}{l}\text { Porto Alegre } \\
\text { (Rio Grande } \\
\text { do Sul) }\end{array}$ & $15-49$ anos & Revisão de dados do SIM e DO & $\begin{array}{c}\text { Aborto e } \\
\text { hipertensão }\end{array}$ & $62,8(1999) / 35,5$ (2001) & \\
\hline $\begin{array}{l}\text { Andrada } \\
\text { et al. } 42\end{array}$ & 2001 & Santa Catarina & $10-49$ anos & $\begin{array}{l}\text { Revisão de } 25 \% \text { óbitos de } \\
\text { mulheres em idade fértil: DO e } \\
\text { fichas de investigação de MIF }\end{array}$ & Hipertensão & $\mathrm{NE}$ & \\
\hline $\begin{array}{l}\text { Saviato } \\
\text { et al. } 43\end{array}$ & $\begin{array}{l}1996- \\
2005\end{array}$ & Santa Catarina & 10-49 anos & $\begin{array}{l}\text { Revisão dos óbitos SIM e } \\
\text { SINASC }\end{array}$ & $\begin{array}{l}\text { Óbitos totais e } \\
\text { por hipertensão }\end{array}$ & $43,3 / 8,6$ * & \\
\hline $\begin{array}{l}\text { Souza } \\
\text { et al. } 44\end{array}$ & $\begin{array}{l}1996- \\
2005\end{array}$ & Santa Catarina & $10-49$ anos & $\begin{array}{c}\text { Revisão dos óbitos SIM e } \\
\text { SINASC }\end{array}$ & $\begin{array}{l}\text { Óbitos por } \\
\text { aborto }\end{array}$ & $1,5 * \star$ & \\
\hline $\begin{array}{l}\text { Soares } \\
\text { et al. } 45\end{array}$ & $\begin{array}{c}1994- \\
1996\end{array}$ & Paraná & 10-49 anos & RAMOS & Hipertensão & 84,6 & \\
\hline $\begin{array}{l}\text { Soares et } \\
\text { al. } 46\end{array}$ & $\begin{array}{l}1991- \\
2005\end{array}$ & Paraná & $10-49$ anos & RAMOS & Hipertensão & $\begin{array}{c}86,4(1991 / 1993) / 64,2 \\
(2003 / 2005)\end{array}$ & \\
\hline Soares 47 & $\begin{array}{l}1997- \\
2005\end{array}$ & Paraná & 10-49 anos & RAMOS & $\begin{array}{l}\text { Óbitos por } \\
\text { hipertensão }\end{array}$ & $\begin{array}{c}13,1 *(1997 / 1999) / 11,8 \\
*(2003 / 2005)\end{array}$ & \\
\hline $\begin{array}{l}\text { Matos et } \\
\text { al. } 48\end{array}$ & $\begin{array}{l}1998- \\
2004\end{array}$ & Paraná & $10-49$ anos & Revisão de dados do SIM: DO & $\begin{array}{l}\text { Óbitos por } \\
\text { aborto }\end{array}$ & $\mathrm{NE}$ & \\
\hline $\begin{array}{l}\text { Souza et } \\
\text { al. } 49\end{array}$ & $\begin{array}{l}2003- \\
2005\end{array}$ & Paraná & $10-49$ anos & $\begin{array}{c}\text { Revisão de dados do Comitê de } \\
\text { Mortalidade Materna e DO }\end{array}$ & $\begin{array}{l}\text { Óbitos por } \\
\text { aborto }\end{array}$ & $64,3 / 3,6 \star \star$ & \\
\hline $\begin{array}{l}\text { Martins \& } \\
\text { Tanaka } 50\end{array}$ & $\begin{array}{l}1993- \\
1998\end{array}$ & Paraná & 10-49 anos & $\begin{array}{c}\text { RAMOS/Banco de dados do } \\
\text { Comitê Estadual de Morte } \\
\text { Materna }\end{array}$ & Hipertensão & Pretas: 342/Brancas: 51 & \\
\hline Martins 16 & $\begin{array}{l}2000- \\
2002\end{array}$ & Paraná & 10-49 anos & $\begin{array}{c}\text { Revisão de dados do SIM } \\
\text { e relatórios do Comitê de } \\
\text { Mortalidade Materna }\end{array}$ & Hipertensão & $\begin{array}{c}\text { Brancas: 49/Pardas: } \\
\text { 213/Pretas: } 407\end{array}$ & \\
\hline
\end{tabular}

(continua) 


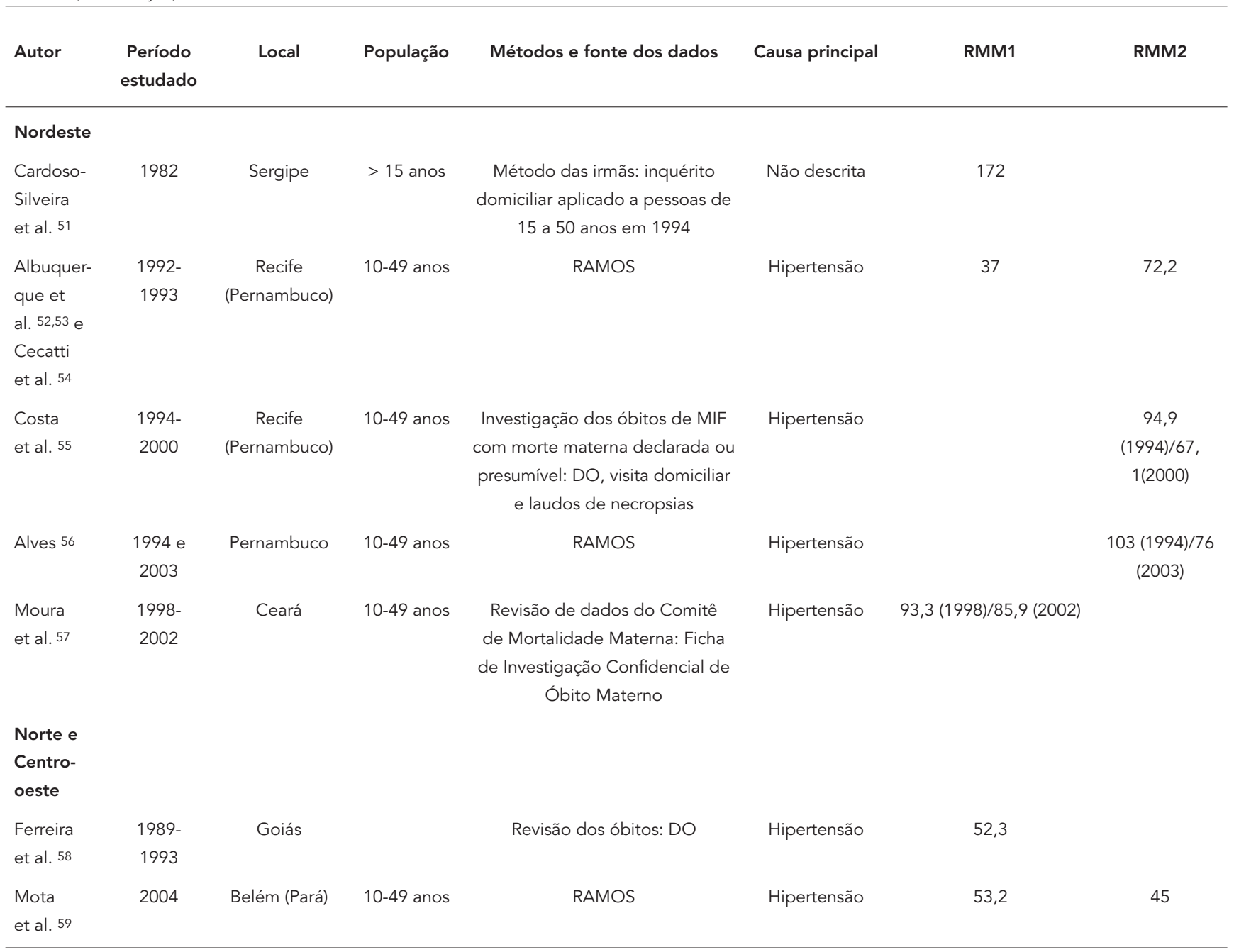

CIS/SEADE: Centro de Informação de Saúde, Fundação Sistema Estadual de Análise de Dados; DF: Distrito Federal; DO: Declaração de Óbito; MIF: mulheres em idade fértil; NE: não estimada; SIH-SUS: Sistema de Informações Hospitalares do SUS; SIM: Sistema de Informações de Mortalidade; SINASC: Sistema de Informações de Nascidos Vivos.

* Somente pelas causas hipertensivas;

** Somente por aborto.

\section{Brasil}

Siqueira et al. ${ }^{10}$, estudaram a mortalidade materna nas regiões, em 1980. As principais causas de óbito foram doenças hipertensivas, hemorragias, e infecções puerperais, seguidas do aborto. Nesse trabalho a RMM foi de 70,3/100.000 NV, ressalvando-se a dificuldade, naquela época, da real estimativa de nascimentos e óbitos maternos. Foi estimada correção da RMM para 154,3/100.000 NV. O grupo etário com o maior RMM foi o de 40-49 anos (RMM não corrigida de 167,3/100.000 $\mathrm{NV}$ ) e a região com o pior indicador foi a Norte (RMM corrigida de 337/100.000 NV). Em estudo da mortalidade na adolescência ${ }^{11}$, foi observada para a faixa de 15-19 anos RMM de 64,3/100.000 NV em 1980.
Victora 12 revisou os óbitos maternos de 1995 a 1997, encontrando RMM de 150 a 200 por 100.000 NV. Esse autor estimou a subnotificação mais elevada entre os estudos brasileiros. A pior situação foi a da Região Nordeste, com RMM de 219/100.000 NV e a relação causas diretas/indiretas foi de 11,3. A melhor situação foi a da Região Sudeste com RMM de 117/100.000 NV e relação causas diretas/indiretas de 3,2.

Sousa et al. 13 , utilizaram os sistemas de informações de dados vitais - Sistema de Informações de Mortalidade (SIM) e Sistema de Informações de Nascidos Vivos (SINASC) - e o Sistema de Informações Hospitalares do SUS (SIH-SUS) para o cálculo da RMM nas capitais e no Distrito Federal, em 2002. No relacionamento com o SINASC, foi possível localizar $46,5 \%$ das mortes maternas 
e, com o SIH-SUS, $55,2 \%$ das mortes. O fator de ajuste para o país como um todo foi 1,4, elevando a RMM de 46,4 para 64,9 por 100.000 NV. A região com a maior RMM foi a Nordeste (95) e a menor foi a Região Centro-oeste (44).

Um estudo em 15 capitais brasileiras 14, realizado em 2002, identificou, após correção de 1,4, uma RMM de 54,3 por $100.000 \mathrm{NV}$, variando entre 42 e 73,2/100.000 NV no Sul e Nordeste, respectivamente. Em outro artigo 15, analisaram-se as causas desses óbitos, identificando as obstétricas diretas como $75 \%$ do total das 201 mortes, com relação de 2,95. Por sua vez, as doenças hipertensivas foram responsáveis por um terço das causas diretas. Mais de $60 \%$ dos óbitos ocorreram no puerpério.

Por último, Martins 16 analisou a mortalidade materna de acordo com a raça, utilizando dados de comitês, relatórios e teses. Concluiu que as mulheres pretas têm as maiores RMM em vários estados e capitais brasileiras, com um risco relativo comparado às brancas variando entre 3,7 (Bahia) e 8,2 (Paraná). Destaca-se o risco aumentado de morte por doenças hipertensivas, tanto crônicas - RMM de 50/100.000 NV, comparado com 2,75 nas brancas - como específicas da gravidez - RMM de 85,7/100.000 NV, comparada à RMM de 10,3 nas brancas.

\section{$\underline{\text { Região Sudeste }}$}

Em 1980, no Estado de São Paulo 10, a primeira causa de óbito materno foi eclampsia, seguida das hemorragias e infecções. A RMM foi 58/100.000 NV, a menor na Região Sudeste no período. A investigação foi estendida até 198417 e persistiram as mesmas causas, porém com abortamento na terceira posição. Houve redução na RMM, que chegou a 48,6/100.000 NV em 1984.

Laurenti 18 realizou um estudo retrospectivo da mortalidade materna no Estado e no Município de São Paulo, de 1960 a 1990. Houve queda da RMM de 144/100.000 NV, na década de 60, para 47,4/100.000 NV na década de 90. Os valores sempre foram superiores no interior do estado, comparados à capital, mas nos anos 90 se igualaram. Ocorreu mudança no perfil das causas na cidade de São Paulo: enquanto na década de 60, as complicações da gravidez e do parto foram semelhantes (32 e 26\%, respectivamente), na década de 90 , as primeiras atingiram $64 \%$ das causas, quando houve queda da segunda para apenas 7\%. Também diminuiu a contribuição do abortamento (de $19 \%$ para $10 \%$ ).

Na década seguinte, Haddad \& Silva ${ }^{19}$, em estudo dos óbitos de mulheres em idade fértil no mesmo estado, de 1991 a 1995, verificaram RMM de $47 / 100.000 \mathrm{NV}$. As principais causas foram as doenças hipertensivas.

Laurenti et al. 20,21, investigando os óbitos de mulheres em idade fértil, com dados de estatísticas vitais, registros hospitalares, necroscópicos e entrevistas domiciliares - método RAMOS - identificaram níveis de subinformação superiores a $100 \%$ e RMM de 99,6/100.000 NV para o Município de São Paulo em 1986.

Outros estudos 22,23,24,25,26,27 nessa mesma cidade se seguiram na década de 90, organizados pelo Comitê Central de Mortalidade Materna. Em todos os anos, houve subnotificação: enquanto a RMM oficial era em torno de 40/100.000 NV, a correção apontava RMM de 50/100.000 NV no período de 1993 a 1995 e próxima de 60/100.000 NV ao final da década. Para o período 1995-1999, realizou-se uma investigação adicional 28, incluindo apenas mulheres cuja causa de óbito foi doença hipertensiva $-23 \%$ dos casos no período. A maioria desses óbitos ocorreu no puerpério, envolvendo acidentes cerebrovasculares e edema agudo de pulmão. Os quadros de eclampsia e pré-eclampsia foram mais comuns nas mulheres jovens enquanto aqueles devidos à doença hipertensiva crônica ocorreram nas mais velhas.

Foram realizados quatro estudos em Campinas, investigando as declarações de óbito de mulheres de 10-49 anos: dois sobre o período 1985-1991 29,30 e dois de 1992-1994 31,32. Foi detectada subnotificação em todos os anos estudados. Houve uma tendência à redução da RMM, que fechou em 1994 com 42/100.000 NV e elevado porcentual de causas obstétricas diretas. Durante os anos de 1985-1991, as doenças hipertensivas predominaram como causa principal. Já no período de 1992-1994, as complicações do aborto suplantaram as demais. Os fatores de risco para óbito materno foram avaliados, comparando-se 62 óbitos ocorridos entre 1985 e 1991 com 248 controles, e o único fator associado foi o parto cesáreo nas mulheres atendidas em serviços públicos.

Por último, em Jundiaí (São Paulo), Matias et al. 33 compararam a mortalidade materna declarada (no SIM) com a investigação pelo método RAMOS, no período de 1999 a 2006. Foram identificadas 12 mortes maternas, sendo seis declaradas e seis não-declaradas. A subnotificação foi de $50 \%$ (fator de ajuste igual a dois). A RMM oficial foi 14,7 e a corrigida de 29,4/100.000 NV. As causas básicas foram reclassificadas na maioria dos casos. As causas obstétricas diretas foram mais prevalentes, dentre elas, eclampsia e síndrome HELLP, seguidas por infecções.

Rezende et al. ${ }^{34}$, em Uberlândia (Minas Gerais), 1997, identificaram predominância de causas diretas, destacando-se a toxemia. A RMM 
corrigida foi 44,4/100.000 NV e, após análise individualizada dos casos, foram detectadas deficiências na assistência pré-natal. A maioria dos óbitos era evitável e as principais falhas foram número inadequado de consultas, grande intervalo entre a última consulta e o parto, e investigação inadequada dos fatores de risco.

No Estado do Rio de Janeiro, o estudo de Silva 35 avaliou o período de 1977-1987, encontrando um declínio na RMM de $46 \%$ (de 111/100.000 NV em 1977 para 56/100.000 NV em 1988), porém tendendo à estabilização. Observou-se, também, uma diferença entre a RMM da cidade do Rio de Janeiro e a dos demais municípios, sendo esta última bem mais elevada. As principais causas foram: doenças hipertensivas, hemorragia e aborto.

Na cidade do Rio de Janeiro 36, analisandose dados de 1993 a 1996, a RMM variou de 74 a 55/100.000 NV, com elevado porcentual de causas obstétricas diretas, sendo a primeira causa a hipertensão. Os maiores valores da RMM se concentraram nas mulheres com idade igual ou superior a 35 anos. Destacou-se a faixa etária acima dos 40, com RMM de 228/100.000 NV. A RMM mostrou associação inversa com a escolaridade, sendo 28/100.000 NV entre as mulheres com nível superior e alcançando 164/100.000 NV entre as analfabetas.

Já Melo \& Knupp 37, estudando o período de 1996-2004, observaram incremento na RMM, que atingiu 69/100.000 NV em 2004. Essas autoras investigaram a distribuição espacial dos óbitos, ficando caracterizada a Zona Oeste da Cidade do Rio de Janeiro como a de mais elevada RMM (acima de 150/100.000 NV) e aquela com maior fluxo para hospitais em outras regiões do município. Em relação ao grupo de causas, predominaram as obstétricas diretas e observou-se que para as mulheres com idade entre 20 e 29 anos o aborto foi a principal causa, não aparecendo em outras faixas, nas quais as doenças hipertensivas foram as principais.

Kale \& Costa 38 , analisaram o período de 2000 a 2003 na cidade do Rio de Janeiro, usando dados do SIM, SINASC e dos Comitês, e encontraram RMM de 59,5/100.000 NV, um pouco mais baixa que a relatada por Melo \& Knupp 37 . As causas obstétricas diretas foram predominantes, com eclampsia e pré-eclampsia em primeiros lugares. Destaca-se a contribuição da síndrome de imunodeficiência adquirida, que foi responsável por um quarto das causas indiretas. Foi observada uma distribuição de escolaridade desfavorável, assim como um maior percentual de óbitos entre mulheres pardas ou pretas.

\section{Região Sul}

Dois estudos avaliaram a mortalidade materna no Estado do Rio Grande do Sul na década de 80: o primeiro, do período de 1979 a 198039 e o segundo, de 1978 a 1988 40. Entre os anos 70 e 80, a RMM estava em torno de $60 / 100.000 \mathrm{NV}$, sendo toxemia a primeira causa, seguida de abortamento provocado. No decorrer da década houve queda da RMM até atingir 20/100.000 NV em 1988. Manteve-se a doença hipertensiva como causa principal. Não há relato de correção dos dados, portanto, a RMM pode estar subestimada.

Riquinho \& Correia 41, na cidade de Porto Alegre (Rio Grande do Sul), encontraram RMM variando entre 62,88/100.000 NV (1999) e $33,55 / 100.000 \mathrm{NV}$ (2001), e as causas mais frequentes de óbito materno foram: infecção pósaborto, hipertensão, infarto agudo do miocárdio, septicemia e causas vinculadas a AIDS.

O Estado de Santa Catarina foi avaliado no ano de 2001 42, sendo reestudados cerca de $25 \%$ dos óbitos de mulheres em idade fértil - apenas aqueles registrados no Comitê de Morte Materna. A causa mais frequente foi hipertensão. $\mathrm{O}$ pré-natal foi deficitário em $33 \%$ dos casos e a informação ausente em outro terço. A RMM não foi calculada, em virtude da deficiência da investigação realizada pelo comitê. Os autores referem que a estimativa corrigida no período estudado foi de $77 / 100.000 \mathrm{NV}$.

Um estudo específico sobre os óbitos maternos por doenças hipertensivas em Santa Catarina abrangeu o período de 1996-2005 43. Com pequenas variações, essa causa foi responsável por $20 \%$ do total de mortes maternas, sendo a eclampsia a principal morbidade nesse grupo. A RMM geral foi 43,3/100.000 NV e a específica por hipertensão 8,6/100.000 NV.

No mesmo período, nesse estado, foram avaliados os óbitos por complicações do aborto 44 . A RMM por essa causa específica foi 1,5/100.000 $\mathrm{NV}$, mas quando analisadas as diferentes regiões do estado, observou-se uma elevada RMM - 5,1/100.000 NV na região oeste. Não foi possível avaliar a escolaridade dessas mulheres pois a informação estava ausente em mais de 50\% dos casos.

No Paraná, o Comitê de Mortalidade Materna estudou o período de 1994-1996 45. A RMM foi de $84,6 / 100.000 \mathrm{NV}$, destacando-se a faixa etária de 45-49 anos, com RMM de 1.161/100.000 NV. As mulheres sem nenhuma escolaridade tiveram RMM de 213,4/100.000 NV, comparadas àquelas com mais de 11 anos de estudos - 34,2/100.000 NV. As causas básicas foram hipertensão, hemorragias e infecção puerperal. As falhas identificadas nos casos de óbito por hipertensão foram 
acompanhamento inadequado no pré-natal e subutilização do sulfato de magnésio na internação. Nos óbitos por hemorragia, houve dificuldade na hemotransfusão em mais de $40 \%$ dos casos.

No mesmo estado, para o período de 1991 a 2005 46, a RMM variou de 86,4/100.000 NV de 1991-1993 para 64,2/100.000 NV no período 2003-2005. A subnotificação se manteve elevada - em torno de $40 \%$ - durante todos os anos estudados. A concentração de subnotificação foi maior nas causas diretas (53\% dos casos). Foi observado que o preenchimento incorreto dos campos 43 e 44 da declaração de óbito ocorreu em 20\% dos óbitos.

Para o período de 1997 a 2005, foram estudados separadamente os óbitos por eclampsia e pré-eclampsia, as causas básicas mais comuns 47 . Observou-se pequena variação na contribuição dessas causas nos anos estudados - cerca de $18 \%$ do total de óbitos maternos. Considerando fatores sociodemográficos, o risco foi seis vezes maior nas mulheres de 40-49 anos, quando comparadas com as de 20-29 anos, e 16 vezes maior entre as mulheres com baixa escolaridade $(<8$ anos) em relação àquelas com mais de 8 anos de estudos. Duas outras pesquisas avaliaram os óbitos maternos por aborto no Paraná 48,49. No período de 1998 a 2004, corresponderam a 6,27\% (51) dos óbitos maternos. Destacou-se um elevado percentual desses óbitos - $23 \%$ - entre as adolescentes 49. Entre 2003-2005, as complicações do aborto também representaram $6 \%$ dos óbitos maternos, com uma RMM específica de 3,6/100.000 NV 49. A infecção pós-aborto foi a principal complicação causadora de óbito e, na análise de evitabilidade, $88 \%$ foram considerados evitáveis. Os abortos foram, em sua maioria, incompletos e provocados. As mulheres jovens, casadas, com baixo status econômico, social e reprodutivo foram as mais atingidas.

Os estudos específicos de Martins 16 e Martins \& Tanaka 50 sobre raça identificaram RMM maior entre mulheres pretas e pardas. Para o Paraná, no período de 1993-1998, os coeficientes de mortalidade materna foram 385,4; 342,3 e 51,6/100.000 NV entre mulheres amarelas, pretas e brancas, respectivamente. Já no período de 2000 a 2002, as mulheres brancas tiveram RMM de $49,2 / 100.000 \mathrm{NV}$, as pardas $213,6 / 100.000 \mathrm{NV}$ e as pretas $407 / 100.000 \mathrm{NV}$.

\section{Região Nordeste}

Em Sergipe 51, foi utilizado o "método das irmãs" para estimar a RMM em 1982, aplicando-se um questionário em 1994 aos habitantes aleatoriamente selecionados para o Diagnóstico de Saú- de Materno-Infantil. Com base nessas respostas sobre número de irmãs e casos de óbito no período gravídico-puerperal, estima-se a RMM retrospectivamente. Considerando os habitantes entre 15 e 50 anos, a RMM foi de 135/100.000 NV em 1982.

Os trabalhos de Albuquerque et al. 52,53 e Cecatti et al. 54 analisam a mortalidade materna em Recife (Pernambuco) no período de 1992-1993, encontrando 22 casos não-notificados. Após correção, a RMM atingiu 72,2/100.000 NV. As principais causas foram doenças hipertensivas, infecção puerperal e aborto. Um terço das mulheres não tinha realizado o pré-natal.

Costa et al. 55, ao estudarem a mortalidade materna de 1994-2000, na mesma cidade, identificaram uma queda na subnotificação, que ficou em torno de $28,7 \%$. A RMM do período esteve em 75,5/100.000 NV, mas houve queda de 94,9/100.000 NV em 1994 para 67,1/100.000 NV em 2000. A hipertensão permaneceu como causa principal, seguida das hemorragias, havendo queda da participação de infecção puerperal e aborto. Foi observado que $22 \%$ dessas mulheres não receberam qualquer cuidado pré-natal. A maioria dos óbitos ocorreu em instituições públicas e mais de $80 \%$ foram considerados evitáveis.

Alves 56 avaliou os padrões da mortalidade materna em três regiões de Pernambuco - metropolitana, agreste e sertão - em 1994 e 2003. Foram incluídas as mortes tardias e a variação foi de 103/100.000 NV em 1994 para 73/100.000 NV em 2003. A pior situação foi a do sertão, que em 2003 ainda contabilizava RMM de 126/100.000 NV. A subnotificação identificada no estudo foi de $46 \%$. Caso fossem considerados apenas os óbitos até 42 dias de puerpério, a RMM seria de 63/100.000 NV para Pernambuco, em 2003. Aproximadamente $94 \%$ das mortes maternas foram evitáveis, destacando-se que $13 \%$ das mulheres não fizeram pré-natal e, entre as que fizeram, foi considerado adequado em apenas $21 \%$. Somente $40 \%$ das mulheres obtiveram atendimento na primeira unidade de saúde procurada.

No Ceará, Moura et al. 57 estudaram o período de 1998 a 2002. A RMM variou de 93,3/100.000 NV no início do período a 85,9 em 2002. Houve subinformação de variáveis como renda, prénatal e escolaridade em percentual elevado dos óbitos.

\section{Regiões Norte e Centro-oeste}

Ferreira et al. 58 estudaram os óbitos maternos em Goiás no período de 1989 a 1993, cuja RMM foi de 52,3/100.000 NV, apresentando variações muito pequenas a cada ano. Predominaram as causas diretas, sendo as principais toxemia e in- 
fecção, seguidas de hemorragias. Deve ser ressaltado que quase $10 \%$ dos casos de infecção foram secundários a abortamento, revelando a contribuição desta causa primária.

Em Belém (Pará), Mota et al. 59 identificaram, entre as mortes maternas, $92,8 \%$ de causas obstétricas diretas, sendo a proporção mais elevada entre os estudos brasileiros apresentados neste trabalho. Embora tenham encontrado uma subnotificação de 50\%, a RMM após a investigação, 45/100.000 NV, foi inferior à razão obtida por meio do SIM, 53,2/100.000 NV. Esse achado pouco comum é explicado pelo fato de o SIM ter registrado óbitos maternos não confirmados pelo estudo, como casos identificados pelo estudo como sendo óbito materno mas que não tinham sido informados ao SIM como tal. Quase $80 \%$ dos óbitos foram considerados evitáveis, sendo a causa mais comum a doença hipertensiva.

\section{Discussão}

Existe um descompasso entre a relevância da mortalidade materna como problema de saúde pública e a quantidade de artigos produzidos sobre o tema no Brasil.

Os estudos publicados estão concentrados na Região Sudeste e são, em sua maioria, de cunho descritivo, com identificação das causas básicas, avançando pouco na investigação dos determinantes dos óbitos maternos.

Até a década de 90, as fontes de dados principais foram os dados primários de declarações de óbito. Mais recentemente, alguns estudos também usaram os dados do SIM, SINASC eSIH-SUS, por meio de relacionamento probabilístico de registros. Essa metodologia tem ampliado as estratégias de identificação das mortes maternas 60,61. Quanto à metodologia usada, vários estudos adotaram o método RAMOS, que tenta superar as limitações do uso isolado da declaração de óbito ${ }^{5}$.

É interessante comparar os estudos de Sousa et al. 13 e Laurenti et al. 14,15, que exploraram a mortalidade materna no mesmo período (2002), com diferentes metodologias. Os valores de RMM não ajustadas foram semelhantes, mais elevados no estudo de Souza et al. 13, e destacando-se o pior desempenho da Região Nordeste. Por outro lado, o uso de diferentes métodos também pode dificultar a comparabilidade dos estudos, como ilustra a diferença na RMM encontrada na cidade do Rio de Janeiro em um mesmo período 37,38 .

A maioria dos estudos confirmou níveis elevados de subnotificação dos óbitos maternos, em torno de 40 a 50\%, assim como informação au- sente nas declarações para variáveis importantes como cor da pele e escolaridade e dos campos 43 e 44.

Essas questões não são exclusivas do Brasil; a medida acurada da mortalidade materna permanece um desafio para outros países em desenvolvimento 62,63 .

Deve ser destacado que a produção científica foi influenciada pela atividade dos Comitês de Morte Materna. Porém o desempenho dos comitês é heterogêneo nas localidades brasileiras, com percentual de investigações dos óbitos de mulheres em idade fértil variando de 45 a $90 \%$, conforme observado nos artigos que se basearam nesses relatórios 38,42,49,50.

Em relação aos achados principais dos estudos aqui revisados, observaram-se variações muito grandes da RMM no Brasil - 39,9/100.000 NV (Jundiaí, 2005) a 85,9/100.000 NV (Ceará, 2002). Deve ser enfatizado que, quando explorados diferenciais dentro dos estados e/ou grandes cidades, essas variações também foram percebidas 37,44. Isso é observado em outros países, mesmo naqueles com valores de RMM mais baixos, como a Argentina, com RMM global em 2002 de 46/100.000 NV, mas, em algumas províncias, alcançando 166/100.000 NV 64. Essas discrepâncias revelam as desigualdades socioeconômicas das regiões e apontam para a necessidade de monitoramento e intervenções diferenciadas, de acordo com as realidades e estimativas locais.

A OMS considera elevada a RMM superior a 25/100.000 NV. Na América Latina, o Chile é um exemplo de país que conseguiu uma redução acentuada - de 40/100.000 NV em 1990 para $18,7 / 100.000 \mathrm{NV}$ em 2000, provavelmente por readequação dos serviços de saúde voltados para a prevenção da mortalidade materna 65,66 . No caso do Brasil, observamos que a RMM vem caindo, mas em velocidade aquém do desejado, e se mantém estagnada nos últimos anos.

As causas diretas tiveram a maior contribuição em todos os períodos e as doenças hipertensivas permanecem como causa principal, secundadas pelas hemorragias. Esse é o padrão relatado para a América Latina, com alguma variação entre as duas causas na primeira posição ${ }^{3}$. No Chile 64, entre 1990-2000, as doenças hipertensivas foram responsáveis por $20,8 \%$ das mortes maternas, apresentando redução ao longo do período estudado. No Equador e na Colômbia, em 2005, alcançaram quase $30 \%$; já na Bolívia, no Peru e na Venezuela, as doenças hipertensivas ficaram em segundo lugar, depois das causas hemorrágicas 67.

Em outros locais também se observou doença hipertensiva como causa principal. Na África do Sul 68, entre 2002 e 2004, essa morbidade repre- 
sentou $19 \%$ do total de causas diretas, e em $50 \%$ dos casos a eclampsia foi o diagnóstico. $\mathrm{Na} \mathrm{Ho-}$ landa, onde a RMM é baixa, variando em torno de 9 a 12/100.000 NV, a pré-eclampsia foi a causa principal de morte 69,70. Segundo Duley 71, $10 \%$ a $15 \%$ das mortes maternas por causa direta em todo o mundo estão associadas à pré-eclampsia e eclampsia. Onde a RMM é alta, o porcentual de mortes atribuíveis à eclampsia é maior que o da pré-eclampsia. Nos estudos brasileiros, muitas vezes essas causas estão agrupadas sob a classificação de doenças hipertensivas, mas quando estratificadas, revela-se a predominância da eclampsia.

As prevalências de hemorragia pós-parto e hemorragia pós-parto severa são estimadas em $6 \%$ e $1,8 \%$ de todos os partos, respectivamente 72 . Esses quadros podem evoluir para óbito materno, sendo a causa principal na África (33,9\%) e na Ásia (30,8\%) 3. Na América Latina, foi a principal causa em países como Bolívia, Peru eVenezuela 67. Nos estudos nacionais, essa causa ocupou o segundo lugar na maioria dos estados, muitas vezes em percentual bem próximo do das doenças hipertensivas.

Já as complicações pós-aborto compareceram em pequeno percentual, $5 \%$ a $15 \%$ na maioria dos estudos, diferentemente de outros países da América Latina, como Uruguai e Argentina, onde podem chegar a $25 \% 64,73$. No entanto, é possível se pensar em uma subestimativa dos dados, por questões legais e culturais que permeiam o aborto no país.

Nas regiões Sudeste e Sul, pode-se traçar a tendência temporal das cidades do Rio de Janeiro e São Paulo e do Estado do Paraná. Esses locais, apesar da atuação dos comitês e do grau de desenvolvimento e recursos da assistência em saúde, têm apresentado, após redução na década de 90, tendência à estagnação do indicador de mortalidade materna.

No Rio de Janeiro, considerando-se a década de 1993 a 2003, a RMM tem se mantido em torno de 60/100.000 NV. Em que pese a melhor investigação dos óbitos nos anos recentes, não se justifica um valor tão elevado da RMM. Houve uma mudança no padrão das causas, com aumento da contribuição das indiretas de $8 \%$ para $17,5 \%$. Em todos os estudos verificou-se a vulnerabilidade social das mulheres que evoluíram para óbito, seja em relação à baixa escolaridade, à raça ou ao acesso aos serviços de saúde.

Quanto à cidade de São Paulo, o comitê paulistano 74, ao analisar o decênio 1993-2002, apontou aumento no primeiro quinquênio, provavelmente por melhora das estimativas, e queda no segundo, no entanto em valores muito reduzidos: A RMM era de 58/100.000 NV ao final da década e atingiu 54/100.000 NV em 2002. Houve queda principalmente das mortes por complicações pós-aborto, mas a contribuição das doenças hipertensivas e hemorragias pouco tem mudado.

No Paraná, também houve redução da RMM, mas os dois últimos triênios estudados (20002002, 2003-2005) mostram estabilização do indicador, em torno de 64/100.000 NV. As mulheres que morreram tinham baixa escolaridade e baixa renda.

Quanto aos fatores de risco para óbito materno, embora poucos estudos tenham aprofundado esta investigação, e não tenha sido empregada a análise multivariada, encontrou-se associação entre RMM e idade, escolaridade, raça e prénatal.

A faixa etária de maior risco é a de mulheres acima dos 40 anos. Na cidade de São Paulo, no período de 1993-2002, as mulheres na faixa de 4044 anos apresentaram RMM de 207/ 100.000 NV e as da faixa de 45-49 anos, RMM de 391/100.000 $\mathrm{NV}$, enquanto a faixa de 20-24 anos teve RMM de 35,9/100.000 NV 74. No Rio de Janeiro - 2000 a 20003 - as mulheres de 40-49 anos tiveram RMM de 283/100.000 NV, comparadas às mulheres na faixa de 20-29 anos, com RMM de 39,7/100.000 NV ${ }^{38}$. Mesmo no Chile, onde a RMM é baixa, para mulheres com mais de 40 anos é de 100/100.000 NV 65. Esse risco aumentado se reproduz na análise da principal causa, doença hipertensiva: o estudo do Paraná mostrou que as mulheres com mais de 40 anos tinham RMM específica para pré-eclampsia/eclampsia de 60,5/100.000 NV, comparadas ao grupo de menor risco (20-29 anos), com RMM de 10,7/100.000 NV 45.

A baixa escolaridade, apesar de problemas na qualidade da informação, se mostrou associada ao óbito materno em grande número de estudos, tanto para RMM global como para causas específicas. Em estudos na África e Ásia, a escolaridade elevada foi um forte fator protetor para mortalidade materna 75,76 .

Quanto à cor da pele/raça, observou-se RMM muito mais elevada nas mulheres de cor parda e preta, quando comparadas às brancas. A questão racial foi também abordada por Chor \& Lima 77, ao revelar dados nacionais nos quais as mulheres pretas, em 2001, tiveram RMM sete vezes maior (275 por $100.000 \mathrm{NV}$ ) do que as mulheres brancas $(43 / 100.000 \mathrm{NV})$ ou pardas (46/100.000 NV). Em estudos realizados nos Estados Unidos 7879, observou-se disparidade na mortalidade materna de acordo com a raça para as causas específicas de óbito - pré-eclampsia/ eclampsia, afecções placentárias e hemorragia pós-parto. Os autores advogaram uma interação entre fatores biológicos, como maior severidade da hipertensão nas mulheres pretas, e questões 
dos serviços de saúde, destacando-se o menor acesso ao pré-natal.

Outro achado importante nos estudos nacionais foi a deficiência na atenção pré-natal, medida pelo número de consultas, relatada em vários estudos. Outras falhas identificadas no pré-natal foram: distância entre a última consulta e o parto, e não identificação de fatores de risco. O estudo de Alves 56 foi o único a usar uma classificação de adequação do pré-natal e ratificou a associação entre o cuidado inadequado e maior mortalidade materna. Em outros países, mesmo naqueles com baixa RMM, tem sido enfatizada a relação entre o padrão de cuidado pré-natal e a morte materna 66,69,70.

Em relação à atenção ao parto, destacaramse como falhas na assistência: a subutilização de sulfato de magnésio nas hipertensas e os entraves para transfusão nos quadros hemorrágicos. Essas tecnologias já são corroboradas, mas sua subutilização é prevalente no Brasil e em outros países 80 . A associação com cesariana foi citada em alguns trabalhos, com a ressalva do viés de seleção, já que pacientes mais graves têm indicação do procedimento.

O estudo dos determinantes da mortalidade materna ainda é falho no Brasil, e segundo GilGonzález et al. ${ }^{81}$, esta lacuna é universal e pode explicar em parte por que a produção científica não tem contribuído para um declínio mais robusto deste agravo.

A análise de evitabilidade dos óbitos, embora por critérios nem sempre explicitados, encontrou porcentuais de óbitos evitáveis sempre elevados, revelando que, a mortalidade materna ainda pode ser reduzida.

Em relação às limitações do estudo, dois pontos merecem discussão. O primeiro, em relação à busca, que não abrangeu teses e relatórios de comitês. Esses trabalhos certamente teriam enriquecido os resultados, mas o estudo se ateve à publicação científica acadêmica e já revisada por comitês editoriais, para avaliar sua contribuição e poder sugerir novas vertentes. Outro motivo para a exclusão das teses foi de ordem prática: não haveria possibilidade de identificar e resgatar todas as teses no tempo previsto para a revisão, que se constituiu na dissertação de mestrado da autora principal.

Não é possível saber se houve viés por conta da exclusão de teses, mas o fato de haver poucos estudos nas regiões Norte, Nordeste e Centrooeste aponta para a baixa produção ou dificuldade em publicar os resultados de teses destes locais.

Em segundo lugar podemos apontar as dificuldades metodológicas decorrentes dos próprios artigos, como o uso de diferentes critérios, que dificultam a comparação entre os estudos e a extração de medidas sumárias.

\section{Considerações finais}

A despeito das inconsistências nos valores da RMM, estes são elevados no Brasil, sugerindo baixa efetividade das políticas públicas para enfrentar esta questão.

A melhoria das condições de saúde reprodutiva é um desafio, já que as situações de risco atingem, principalmente, mulheres com pouco acesso aos serviços de saúde. Esse fato exige uma assistência ao pré-natal e ao parto de qualidade, um controle social eficaz com ampliação e qualificação dos Comitês de Morte Materna e a mobilização de gestores, profissionais de saúde e da sociedade civil na promoção de políticas públicas que busquem a redução da mortalidade materna.

Quanto às futuras pesquisas sobre o tema, urge ampliar a produção científica em regiões pouco estudadas, ou incentivar a publicação de teses realizadas nestes locais. Também deve ser ampliado o estudo dos determinantes da mortalidade materna, com especial foco nos grupos mais vulneráveis, tornando a produção científica ainda mais relevante no enfrentamento deste grave evento entre as mulheres brasileiras. 


\section{Resumo}

O objetivo deste trabalho foi descrever o perfil epidemiológico e a tendência da mortalidade materna no Brasil, por meio de revisão de estudos sobre o tema. Foi realizada busca eletrônica de artigos científicos publicados entre 1980 e 2010, nas bases de dados LILACS e MEDLINE. Identificaram-se inicialmente 486 artigos. Após aplicação dos critérios de inclusão e exclusão restaram 50 artigos. A comparação dos dados mostrou queda da RMM de forma diferenciada nas regiões brasileiras. Os estudos sobre determinação do óbito materno apontaram desigualdades sociais relacionadas à cor da pele e escolaridade. O preenchimento incompleto da declaração de óbito e a subnotificação ainda persistem. Prevaleceram as causas obstétricas diretas, com predomínio das doenças hipertensivas. Quando analisada, a evitabilidade apontou falhas na assistência pré-natal e ao parto. Apesar de sua relevância, são poucos os artigos sobre mortalidade materna no Brasil. A RMM, embora em declínio, permanece em níveis elevados. Melhorias na qualidade da assistência prénatal e ao parto são necessárias.

Mortalidade Materna; Coeficiente de Mortalidade; Revisão

\section{Colaboradores}

M. L. Morse e S. C. Fonseca participaram da concepção e desenho, revisão sistemática da literatura, sistematização dos resultados, sua análise e interpretação, e redação das versões inicial e final do artigo. M. D. Barbosa, M. B. Calil e F. P. C. Eyer participaram na busca dos artigos de interesse, sistematizaram os resultados e contribuíram na redação da versão inicial do artigo.

\section{Agradecimentos}

Os autores agradecem a Virgínia Mariz, da Biblioteca do Instituto de Medicina Integral Professor Fernando Figueira (IMIP); a Mara Regina Sampaio, da Biblioteca da Maternidade Escola da Universidade Federal do Rio de Janeiro (UFRJ) e a toda equipe da Biblioteca da Faculdade de Medicina da Universidade Federal Fluminense (UFF), que atenderam gentilmente às nossas solicitações enviando os artigos necessários para a realização desta revisão.

\section{Referências}

1. World Health Organization/United Nations Children's Fund/United Nations Population Fund/World Bank. Trends in maternal mortality: 1990 to 2008. Estimates developed by WHO, UNICEF, UNFPA and The World Bank. September 2010. http://whqlibdoc.who.int/ publications/2010/9789241500265_eng.pdf (acessado em Out/2010).

2. Pan American Health Organization. Health situation in the Americas: basic indicators 2008. Washington DC: Pan American Health Organization; 2008.

3. Khan K, Wojdyla D, Say L, Gülmezoglu AM, Van Look PF. WHO analysis of causes of maternal death: a systematic review. Lancet 2006; 367:1066-74.

4. World Health Organization. The Millennium Development Goals Report 2008. http://www.un.org/ millenniumgoals (acessado em Jan/2010).

5. Departamento de Ações Programáticas Estratégicas, Secretaria de Atenção à Saúde, Ministério da Saúde. Política nacional de atenção integral à saúde da mulher: princípios e diretrizes. Brasília: Ministério da Saúde; 2004.

6. Tanaka ACD, Mitsuiki L. Estudo da magnitude da mortalidade materna em 15 cidades. São Paulo: Fundo das Nações Unidas para a Infância; 1999.
7. Laurenti R, Mello-Jorge MH, Gotlieb SLD. Mortes maternas no Brasil: análise do preenchimento de variável da declaração de óbito. Inf Epidemiol SUS 2000; (9):43-50.

8. Secretaria de Atenção à Saúde, Ministério da Saúde. Estudo da mortalidade de mulheres de 10 a 49 anos, com ênfase na mortalidade materna: relatório final. Brasília: Editora do Ministério da Saúde; 2006.

9. Ministério da Saúde. Manual dos Comitês de Mortalidade Materna. http://portal.saude.gov.br/portal/ comites_mortalidade_materna_M.pdf (acessado em Jan/2009).

10. Siqueira AAF, Tanaka ACA, Santana RM, Almeida PAM. Mortalidade materna no Brasil, 1980. Rev Saúde Pública 1984; 18:448-65.

11. Siqueira AAF, Tanaka ACA. Mortalidade na adolescência com especial referência à mortalidade materna, Brasil, 1980. Rev Saúde Pública 1986; 20:274-9.

12. Victora CG. Intervenções para reduzir a mortalidade infantil pré-escolar e materna no Brasil. Rev Bras Epidemiol 2001; 4:3-69.

13. Sousa MH, Cecatti JG, Hardy EE, Serruya SJ. Morte materna declarada e o relacionamento de sistemas de informações em saúde. Rev Saúde Pública 2007; 41:181-9. 
14. Laurenti R, Jorge MH, Gotlieb SL. A mortalidade materna nas capitais brasileiras: algumas características e estimativa de um fator de ajuste. Rev Bras Epidemiol 2004; 7:449-60.

15. Laurenti R, Mello Jorge MHP, Gotlieb SLD. Mortes maternas e mortes por causas maternas. Epidemiol Serv Saúde 2008; 17:283-92.

16. Martins AL. Mortalidade materna de mulheres negras no Brasil. Cad Saúde Pública 2006; 22:2473-9.

17. Tanaka ACA, Siqueira AAF, Bafile PN. Situação de saúde materna e perinatal no Estado de São Paulo, Brasil. Rev Saúde Pública 1989; 23:67-75.

18. Laurenti R. A mortalidade materna em áreas urbanas na América Latina: o caso de São Paulo, Brasil. Bol Oficina Sanit Panam 1994; 116:18-26.

19. Haddad N, Silva MB. Mortalidade feminina em idade reprodutiva no Estado de São Paulo, Brasil, 1991-1995: causas básicas de óbito e mortalidade materna. Rev Saúde Pública 2000; 34:64-70.

20. Laurenti R, Buchalla CM, Lolio CA, Santo AH, Mello Jorge MH. Mortalidade de mulheres em idade fértil no Município de São Paulo (Brasil), 1986. I - metodologia e resultados gerais. Rev Saúde Pública 1990; 24:128-33.

21. Laurenti R, Buchalla CM, Lolio CA, Santo AH, Mello Jorge MH. Mortalidade de mulheres em idade fértil no Município de São Paulo (Brasil), 1986. II - mortes por causas maternas. Rev Saúde Pública 1990; 24:468-72.

22. Boyaciyan K, Ferrigno PA, Vega CEP, Barbosa SA, Pazero LC. Mortalidade materna na Cidade de São Paulo de 1993 a 1995. Rev Bras Ginecol Obstet 1998; 20:13-8.

23. Marcus PAF, Vega CEP, Boyaciyan K, Barbosa SA. Estudo da mortalidade materna no Município de São Paulo durante o ano de 1995. Rev Bras Ginecol Obstet 1997; 18:731-6.

24. Pazero LC, Marcus PAF, Vega CEP, Boyaciyan K, Barbosa SA. Estudo da mortalidade materna no Município de São Paulo durante o ano de 1996. Rev Bras Ginecol Obstet 1998; 20:395-403.

25. Vega CEP, Marcus PAF, Pazero LC, Boyaciyan K, Barbosa SA. Estudo da mortalidade materna no Município de São Paulo durante o ano de 1997. Rev Ginecol Obstet 2001; 12:187-97.

26. Pazero LC, Vega CEP, Marcus PAF, Barbosa SA, Boyaciyan K Estudo da mortalidade materna no Município de São Paulo durante o ano de 1998. Rev Ginecol Obstet 2002; 13:198-207.

27. Vega CEP, Marcus PAF, Pazero LC, Boyaciyan K, Barbosa SA. Estudo da mortalidade materna no Município de São Paulo durante o ano de 1999. Rev Ginecol Obstet 2003; 14:52-9.

28. Vega CEP, Kahhale S, Zugaib M. Maternal mortality due to arterial hypertension in São Paulo City (1995-1999). Clinics 2007; 62:679-84.

29. Cecatti JG, Faúndes A, Surita FG. Maternal mortality in Campinas: evolution, under-registration and avoidance. São Paulo Med J 1999; 117:5-12.

30. Cecatti JG, Faúndes A, Parpinelli MA, Surita FG, Amaral E. Fatores associados à mortalidade materna em Campinas, Estado de Sâo Paulo. Rev Ciênc Méd Campinas 2003; 12:39-48.
31. Parpinelli MA, Surita FGC, Pereira BG, Cecatti JG. Mortalidade materna na cidade de Campinas, no período de 1992 a 1994. Rev Bras Ginecol Obstet 1999; 21:227-32.

32. Parpinelli MA, Faúndes A, Cecatti JG, Surita FGC, Pereira BG, Passini Júnior R, et al. Subnotificação da mortalidade materna em Campinas: 1992 a 1994. Rev Bras Ginecol Obstet 2000; 22:27-32.

33. Matias JP, Parpinelli MA, Nunes MKV, Surita FGC, Cecatti JC. Comparação entre dois métodos para investigação da mortalidade materna em município do Sudeste brasileiro. Rev Bras Ginecol Obstet 2009; 31:559-65.

34. Rezende CHA, Moreli D, Rezende IMAA. Mortalidade materna em cidade de médio porte, Brasil, 1997. Rev Saúde Publica 2000; 34:323-8.

35. Silva KS. Mortalidade materna: avaliação da situação no Rio de Janeiro, no período de 1977 a 1987. Cad Saúde Pública 1992; 8:442-53.

36. Theme-Filha MM, da Silva R, Noronha CP. Mortalidade materna no Município do Rio de Janeiro, 1993 a 1996. Cad Saúde Pública 1999; 15:397-403.

37. Melo ECP, Knupp VMAO. Mortalidade materna no Município do Rio de Janeiro: magnitude e distribuição. Esc Anna Nery Rev Enferm 2008; 12:773-9.

38. Kale P, Costa AJL. Maternal deaths in the city of Rio de Janeiro, Brazil, 2000-2003. J Health Popul Nutr 2009; 27:794-801.

39. Madi JM. A mortalidade materna no Estado do Rio Grande do Sul, período 1979 a 1982: análise das principais causas e de suas incidências nas diferentes faixas etárias. Femina 1987; 15:633-9.

40. Ramos JGL, Costa SM, Rosa Jr. A, Jiuliani RP, Corso MA. Mortalidade materna geral e por hipertensão arterial no Estado do Rio Grande do Sul: uma análise de 11 anos (1978-1988). Rev Bras Ginecol Obstet 1995; 17:123-9.

41. Riquinho DL, Correia SG. Mortalidade materna: perfil sócio-demográfico e causal. Rev Bras Enferm 2006; 59:303-7.

42. Andrada AAC, Vitorello DA, Saab Neto JA. Mortalidade materna no Estado de Santa Catarina durante o ano de 2001. ACM Arq Catar Medicina 2003; 32:56-64.

43. Saviato B, Knobel R, Moraes CA, Tonon D. Morte materna por hipertensão no Estado de Santa Catarina. ACM Arq Catar Medicina 2008; 37:16-9

44. Souza ML, Ferreira LAP, Burgardt D, Monticelli M, Bub MBC. Mortalidade por aborto no Estado de Santa Catarina-1996 A 2005. Esc Anna Nery Rev Enferm 2008; 12:735-40.

45. Soares HB, Soares VMN, Carzino E, Araujo CR. Mortalidade materna no Paraná, do anonimato a ação: relatório trienal, 1994-1996. Rev Ginecol Obstet 1998; 9:70-81.

46. Soares VMN, Azevedo EMM, Watanabe TL. Subnotificação da mortalidade materna no Estado do Paraná, Brasil: 1991-2005. Cad Saúde Pública 2008; 24:2418-26.

47. Soares VMN. Mortalidade materna por préeclâmpsia/eclâmpsia em um estado do Sul do Brasil. Rev Bras Ginecol Obstet 2009; 31:566-73. 
48. Matos JC, Luz GS, Pelloso SM, Carvalho MDB. Mortalidade por aborto no Estado do Paraná: 1998 a 2004. Rev Eletrônica Enferm 2007; 9(3). http:// www.fen.ufg.br/revista/v9/n3/v9n3a19.htm.

49. Souza KV, Almedia MRCB, Soares VMN. Perfil da mortalidade materna por aborto no Paraná: 20032005. Esc Anna Nery Rev Enferm 2008; 12:741-9.

50. Martins AL, Tanaka ACA. Mulheres negras e mortalidade materna no Estado do Paraná, 1993 a 1998. Rev Bras Crescimento Desenvolv Hum 2000; 10:27-38.

51. Cardoso-Silveira JA, Cecatti JG, Palma MM, Batista Filho M. Mortalidade materna pelo "Método das Irmãs" no Estado de Sergipe, Brasil. Revista IMIP 1996; 10:97-102.

52. Albuquerque RM, Cecatti JG, Hardy E, Faúndes A. Mortalidade materna em Recife. 1. Avaliação da subenumeração de estatísticas oficiais. Cad Saúde Pública 1997; 13:59-65.

53. Cecatti JG, Albuquerque RM, Hardy E, Faúndes A. Mortalidade materna em Recife: causas de óbitos maternos. Rev Bras Ginecol Obstet 1998; 20:7-11.

54. Albuquerque RM, Cecatti JG, Hardy E, Faúndes A. Fatores sócio-demográficos e de assistência médica associados ao óbito materno. Rev Bras Ginecol Obstet 1998; 20:181-5.

55. Costa AAR, Ribas MSSS, Amorim MMR, Santos LC. Mortalidade materna na Cidade do Recife. Rev Bras Ginecol Obstet 2002; 24:455-62.

56. Alves SV. Maternal mortality in Pernambuco, Brazil: what has changed in ten years? Reprod Health Matters 2007; 15:134-44.

57. Moura E, Ponte M, Silva R, Lopes M. Mortalidade materna no Ceará-Brasil (1998-2002): estudo retrospectivo. Cogitare Enferm 2007; 12:44-51.

58. Ferreira RG, Lima EL, Viggiano MGC, Moreira SF, Lopes MA, Moraes CA. Mortalidade materna no Estado de Goiás. GO Atual 1996; 9:13-6.

59. Mota SMM, Gama SGN, Theme-Filha MM. Mortalidade materna no Município de Belém, Estado do Pará, em 2004: uma avaliação do Sistema de Informações sobre Mortalidade. Epidemiol Serv Saúde 2008; 17:33-42.

60. Almeida MTC, Beltrão KI, Leite IC. Vigilância da mortalidade materna: a identificação de mortes durante o ciclo gravídico puerperal ampliado. Cad Saúde Colet (Rio J.) 2006; 14:265-82.

61. Gomes FA, Mamede MV, Costa Junior ML, Nakano MAS. Morte materna mascarada; um caminho para sua identificação. Acta Paul Enferm 2006; 19:387-93.

62. Hill K, Queiroz BL, Wong L, Plata J, Del Popolo F, Rosales J, et al. Estimating pregnancy-related mortality from census data: experience in Latin America. Bull World Health Organ 2009; 87:288-95.

63. Munjania SP. Joining the dots: a plea for precise estimates of the maternal mortality ratio in subSahara Africa. BJOG 2009; 116 Suppl 1:7-10.

64. Ramos S, Karolinski A, Romero M, Mercer R; Maternal Mortality in Argentina Study Group. A comprehensive assessment of maternal deaths in Argentina: translating multicentre collaborative research into action. Bull World Health Organ 2007; 85:615-22.

65. Donoso SE. Reducción de la mortalidad materna en Chile de 1990 a 2000. Rev Panam Salud Pública 2004; 15:326-30.
66. Ruiz-Rodriguez M, Wirtz VJ, Nigenda G. Organizational elements of health service related to a reduction in maternal mortality: the cases of Chile and Colombia. Health Policy 2009; 90:149-55.

67. Faneite P. Mortalidad materna en la región bolivariana de Latinoamérica: área crítica. Rev Obstet Ginecol Venezuela 2008; 6818-24.

68. Moodley J. Maternal deaths due to hypertensive disorders in pregnancy: Saving Mothers Report 2002-2004. Cardiovasc J Afr 2007; 18:358-61.

69. Schutte JM, Steegers EA, Schuitemaker NW, Santema JG, de Boer K, Pel M, et al. Rise in maternal mortality in the Netherlands. BJOG 2009; 117:399-406.

70. Schutte JM, Schuitemaker NW, van Roosmalen J, Steegers EA; Dutch Maternal Mortality Committee. Substandard care in maternal mortality due to hypertensive disease in pregnancy in the Netherlands. BJOG 2008; 115:732-6.

71. Duley L. The global impact of pre-eclampsia and eclampsia. Semin Perinatol 2009; 33:130-7.

72. Carroli G, Cuesta C, Abalos E, Gulmezoglu AM Epidemiology of postpartum haemorrhage: a systematic review. Best Pract Res Clin Obstet Gynaecol 2008; 22:999-1012.

73. Briozzo L, Vidiella G, Rodríguez F, Gorgoroso M, Faúndes A, Pons JE. A risk reduction strategy to prevent maternal deaths associated with unsafe abortion. Int J Gynaecol Obstet 2006; 95:221-6.

74. Comitê de Mortalidade Materna do Município de São Paulo. Estudo de casos de mortalidade materna do Município de São Paulo durante o decênio 1993-2002. http://www.prefeitura.sp.gov.br/arqui vos/secretarias/compilado_10anos.pdf (acessado em Jan/2009).

75. Alvarez JL, Gil R, Hernández V, Gil A. Factors associated with maternal mortality in Sub-Saharan Africa: an ecological study. BMC Public Health 2009; 9:462

76. Chowdhury ME, Ahmed A, Kalim N, Koblinsky M. Causes of maternal mortality decline in Matlab, Bangladesh. J Health Popul Nutr 2009; 27:108-23.

77. Chor D, Lima CRA. Aspectos epidemiológicos das desigualdades raciais em saúde no Brasil. Cad Saúde Pública 2005; 21:1586-94.

78. Tucker MJ, Berg CJ, Callaghan WM, Hsia J. The Black-White disparity in pregnancy-related mortality from 5 conditions: differences in prevalence and case-fatality rates. Am J Public Health 2007; 97:247-51.

79. Harper M, Dugan E, Espeland M, Martinez-Borges A, McQuellon C. Why African-American women are at greater risk for pregnancy-related death? Ann Epidemiol 2007; 17:180-5.

80. Tsu VD, Coffey PS. New and underutilized technologies to reduce maternal mortality and morbidity: what progress have me made since Bellagio 2003? BJOG 2009; 116:247-56.

81. Gil-González D, Carrasco-Portino M, Ruiz MT Knowledge gaps in scientific literature on maternal mortality: a systematic review. Bull World Health Organ 2006; 84:903-8.

Recebido em 07/Abr/2010 Versão final reapresentada em 16/Nov/2010 Aprovado em 07/Fev/2011 